\title{
Ultrafast mapping of relaxation dynamics of ethylene cation
}

\author{
Matteo Lucchini ${ }^{1,2,}{ }^{*}$, , Mario Murari ${ }^{1,2}$, Giacinto D. Lucarelli ${ }^{1}$, Fabio Frassetto ${ }^{3}$, Luca \\ Poletto $^{3}$, and Mauro Nisoli ${ }^{1,2}$ \\ ${ }^{1}$ Department of Physics, Politecnico di Milano, 20133 Milano, Italy \\ ${ }^{2}$ Institute for Photonics and Nanotechnologies, IFN-CNR, 20133 Milano, Italy \\ ${ }^{3}$ Institute for Photonics and Nanotechnologies, IFN-CNR, 35131 Padova, Italy
}

\begin{abstract}
The complex ultrafast molecular relaxation dynamics of ethylene, initiated by tunable vacuum-ultraviolet $\sim 10$-fs pulses, was measured. Exploiting state selectivity, an unprecedented time-energy mapping of the process was demonstrated on a few-femtosecond temporal scale.
\end{abstract}

\section{Introduction}

The dynamics unfolding on the first few femtoseconds after optical excitation eventually define the photochemistry of many molecular systems $[1,2]$. Following these ultrafast dynamics in real time constitutes a formidable task both experimentally and theoretically. As such, most of them remain unexplored despite their importance. This is the case also for a prototype organic molecule like ethylene, where the exact relaxation dynamics of its cation $\mathrm{C}_{2} \mathrm{H}_{4}{ }^{+}$(the simplest $\pi$ radical) are not completely understood [3, 4].

Here we use few-femtosecond vacuum-ultraviolet (VUV) pulses produced by highorder harmonic generation (HHG) in combination with a time-delay compensated monochromator (TDCM) $[5,6]$ to selectively excite the first four states of ethylene cation. The relaxation dynamics is subsequently interviewed in a pump-probe fashion by a 15 -fs infrared (IR) pulse, which induces molecular fragmentation. A detailed analysis of the temporal evolution of ion yields allows us to pinpoint the path followed by the molecule after excitation, sheading a light on the ultrafast coupling between the internal degrees of freedom and the conical intersections (CIs) which mediate the ultrafast population transfer between the cationic states.

\section{Results and discussion}

15 -fs IR pulses with central wavelength of $\sim 800 \mathrm{~nm}$ and energy of $0.65 \mathrm{~mJ}$ are focused in a gas cell filled with Xe atoms. The comb of odd harmonics generated by HHG is then sent into a TDCM, which is used to select only one harmonic. The TDCM consists of two sections working in a subtractive configuration to compensate for the temporal and spectral

\footnotetext{
* Corresponding author: matteo.lucchini@polimi.it
} 
dispersion. As a result, it is possible to select a single harmonic, while preserving its original temporal duration $[5,6]$.
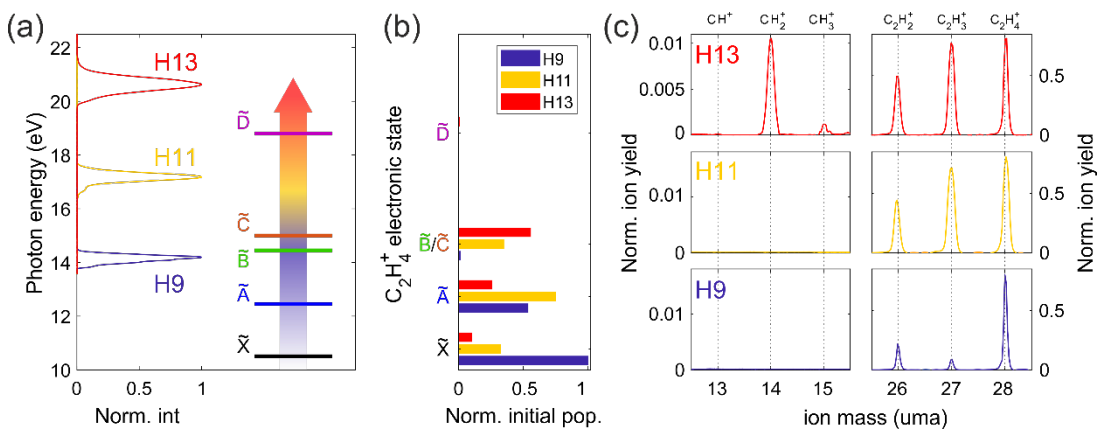

Fig. 1. (a) VUV spectrum of the high-order harmonics used in the experiment together with the first five states of molecular cation [7]. (b) Initial state population calculated with the partial cross-sections taken from [8] and the harmonic spectra in (a). (c) Normalized ion yield of the main charge particles obtained by photo-fragmentation.

After selection, the VUV radiation is focused on an ethylene gas target placed in the focal spot of a time-of-flight (TOF) mass spectrometer. Upon absorption of a harmonic photon, the molecule is ionized and left in an excited state. In particular, we used the $9^{\text {th }}$, the $11^{\text {th }}$ and the $13^{\text {th }}$ harmonic (H9 to H13 in Fig. 1(a)) to selectively populate the first four excited states of $\mathrm{C}_{2} \mathrm{H}_{4}^{+}: \tilde{\mathrm{X}}, \tilde{\mathrm{A}}, \tilde{\mathrm{B}}$ and $\tilde{\mathrm{C}}$ (see Fig. 1(b)). H9 enables a very efficient excitation of the $\tilde{X}$ state, $\mathrm{H} 11$ of the $\tilde{A}$ state, while $\mathrm{H} 13$ predominantly excite the $\tilde{\mathrm{B}}$ and $\tilde{\mathrm{C}}$ states. We estimated the time duration of each harmonic from a cross-correlation photoelectron experiment. We found that the time duration of the harmonic radiation ranges from 12 fs with $\mathrm{H} 9$ to 7 fs with H13.

The internal relaxation process that follows photoexcitation can eventually lead to dissociation. Figure 1(c) shows the main fragments produced by photoexcitation with the VUV pulses. As a consequence of the selective excitation, the fragment yields pertaining to different harmonics qualitatively differ from each other. In order to study the ultrafast relaxation dynamics of $\mathrm{C}_{2} \mathrm{H}_{4}{ }^{+}, \mathrm{VUV}$-pump/IR-probe measurements were performed; the temporal evolution of the ion yields were measured as a function of the relative delay between the two pulses. We point out that the interaction of the molecular ion with the IR pulses can determine remarkable modifications of the relaxation process, thus deviating the molecule from its natural relaxation path and ultimately leading to a change in the fragmentation process. Therefore, the transient features in the delay-dependent ion yields contain a fingerprint of the ultrafast molecular dynamics followed by ethylene after ionization. Figure 2 shows the main results obtained with the three harmonics.

The dynamics observed in the case of H13 excitation (Fig. 2(c)) unfold within the first 50 fs after ionization and are associated with a process of $\mathrm{H} / \mathrm{H} 2$ loss that is proposed to happen on the cation ground state [4], reached by the molecule in about 25 fs (corresponding to the minimum in the $\mathrm{C}_{2} \mathrm{H}_{4}{ }^{+}$yield of Fig. 2(c)). This relaxation to the state $\tilde{\mathrm{X}}$ is characterized by ultrafast nuclear dynamics, which involve mechanism like twisting, pyramidalization and isomerization $[9,10]$, far from being completely understood. In particular, the precise nature and location of the CIs between the different potential energy surfaces that mediate the process remains debated. Our results pave the way for a complete mapping of such CIs. Indeed, the observation of the same bleaching dynamics of $\mathrm{C}_{2} \mathrm{H}_{4}{ }^{+}$for 
all the three harmonics used in the experiments, proves that the bottleneck for the population transfer lies between the $\mathrm{X}^{\tilde{x}}$ and the $\tilde{\mathrm{A}}$ states. The different ultrafast dynamics observed in the $\mathrm{C}_{2} \mathrm{H}_{3}{ }^{+}$and $\mathrm{C}_{2} \mathrm{H}_{2}{ }^{+}$channels, instead, question the exact location of the CIs leading to the loss of one or two $\mathrm{H}$ atoms.

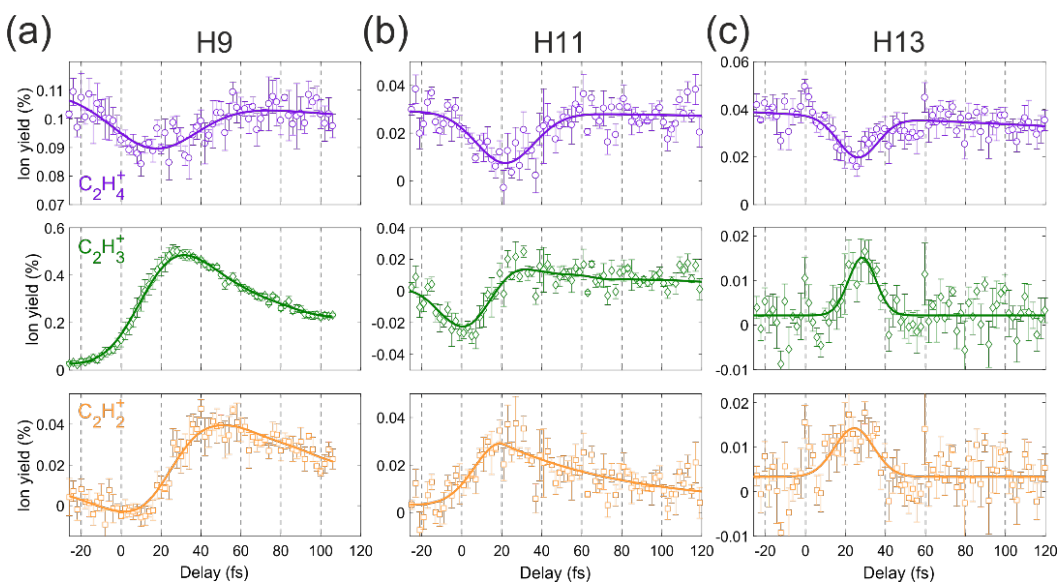

Fig. 2. Delay-dependent IR-induced relative change of the ion yields for $\mathrm{C}_{2} \mathrm{H}_{4}{ }^{+}$(violet circles), $\mathrm{C}_{2} \mathrm{H}_{3}{ }^{+}$ (green diamonds) and $\mathrm{C}_{2} \mathrm{H}_{2}^{+}$(orange squares). (a)-(c) excitation with $\mathrm{H} 9, \mathrm{H} 11$ and $\mathrm{H} 13$, respectively. The error bars represent the error of the mean obtained by summing 4 independent scans for each ion yield. The solid lines are a guide for the eye.

In conclusion, the combination of selective excitation and high time resolution allowed us to disentangle the complex dynamics that follow photoexcitation of ethylene and shed new light onto the internal relaxation process of small organic molecules.

\section{References}

1. D. Polli, et al., Nature 467, 440-443 (2010)

2. M. C. E. Galbraith, et al., Nat. Comm. 8, 1018 (2017)

3. B. Joalland, T. Mori, T. J. Martínez and A. G. Suits, J. Phys. Chem. Lett. 5, 1467-1471 (2014)

4. A. Ludwig, et al. J. Phys. Chem. Lett. 7, 1901-1906 (2016)

5. L. Poletto, P. Villoresi, E. Benedetti, F. Ferrari, S. Stagira, G. Sansone and M. Nisoli, Opt. Lett. 117, 2897-2899 (2007)

6. L. Poletto, F. Frassetto and P. Villoresi, IEEE Journal of Selected Topics in Quantum Electronics 18, 467-478 (2012)

7. R. A. Mackie, et al., Int J Mass Spectrom. 223-224, 67-79 (2003)

8. J. Berkowitz, Atomic and Molecular Photoabsorption, (Academic Press, Amsterdam, 2015)

9. H. Tao, et al., J. Chem. Phys. 134, 244306 (2011)

10. T. K. Allison, et al., J. Chem. Phys. 136, 124317 (2012) 